\title{
GRUPO DE CONVIVÊNCIA COM PESSOAS COM DOENÇA PULMONAR OBSTRUTIVA CRÔNICA: SENTIMENTOS E EXPECTATIVAS ${ }^{1}$ JOINT LIVING GROUP WITH PEOPLE WITH CHRONIC OBSTRUCTIVE PULMONARY DISEASE: FEELINGS AND EXPECTATIONS GRUPO DE CONVIVENCIA CON PERSONAS QUE TIENEN ENFERMEDAD PULMONAR OBSTRUCTIVA CRÓNICA: SENTIMIENTOS Y EXPECTATIVAS
}

\author{
Edilaine Kerkoski², Miriam Süsskind Borenstein ${ }^{3}$, Luciana de Oliveira Gonçalves ${ }^{4}$, Fabiane Ferreira Francioni ${ }^{5}$
}

${ }^{1}$ Trabalho elaborado a partir da prática assistencial referente ao Mestrado em Enfermagem do Programa de Pós Graduação (PEN) da Universidade Federal de Santa Catarina (UFSC), sob a Orientação da Professora Doutora Miriam Süsskind Borenstein.

${ }^{2}$ Mestranda do PEN/UFSC. Professora do Curso de Graduação em Fisioterapia da Universidade do Vale do Itajaí (UNIVALI), Campus Itajaí, SC. Membro do Grupo de Estudos de História do Conhecimento de Enfermagem (GEHCE). Membro do Núcleo de Estudos e Assistência de Enfermagem às Pessoas em Situação Crônica de Saúde (NUCRON).

${ }^{3}$ Doutora em Filosofia da Enfermagem pela UFSC. Professora Adjunto do Departamento de Enfermagem da UFSC. Coordenadora do GEHCE. Integrante do NUCRON. Pesquisadora do Conselho Nacional de Desenvolvimento Científico e Tecnológico (CNPq).

${ }^{4}$ Mestre em Engenharia de Produção com área de concentração em Ergonomia. Professora do Curso de Graduação em Fisioterapia da UNIVALI. Doutoranda do PEN/UFSC.

${ }^{5}$ Enfermeira. Mestre em Enfermagem. Doutoranda do PEN/UFSC. Professora Substituta do Departamento de Enfermagem da UFSC. Integrante do NUCRON. Bolsista do CNPq.

PALAVRAS-CHAVE: Modalidades de fisioterapia. Educação em saúde.Qualidade de vida.

KEYWORDS: Physical therapy modalities. Health education. Quality of life.

PALABRAS CLAVE: Modalidades de terapia física. Educación en salud. Calidad de vida.
RESUMO: Trata-se de uma pesquisa qualitativa realizada com pessoas com Doença Pulmonar Obstrutiva Crônica em uma Clínica de Fisioterapia da Universidade do Vale do Itajaí/SC. Teve como objetivo identificar sentimentos dessas pessoas relacionados ao seu problema de saúde. Para tanto, foi realizado um grupo de convivência com onze pacientes e um familiar. A partir da análise de dados emergiram três categorias distintas: a tristeza e sofrimento ocasionados pela doença; as limitações e incapacidades físicas no cotidiano e o desejo de melhora em relação ao quadro clínico estabelecido pela doença. Observamos, portanto, que o grupo de convivência é um espaço importante para pessoas com doenças crônicas, no sentido de provocar mudanças de comportamento, adquirindo novos hábitos saudáveis de vida, também servindo como mola propulsora para que estas pessoas possam expressar sentimentos, contribuindo para melhorar sua qualidade de vida.

ABSTRACT: This study reports qualitative research carried out with people with Chronic Obstructive Pulmonary Disease at a Physiotherapy Clinic of the Itajaí Valley University (Universidade do Vale do Itajaí), Santa Catarina, Brazil. Its objective is to identify the feelings of these people concerning their health problem. In order to do so, a joint living group with eleven patients' and one parent was carried out. From the data analysis, three different categories emerged: unhappiness and suffering caused by the disease; the limitations and physical incapacity everyday, and the desire for improvement with regard to the clinical status established for the disease. We observed, however, that a joint living group is an important opportunity for people with chronic disease to stimulate behavioral change, new healthful habits of life, also acting as an inspirational spring for these people to express feelings to help get better their quality life.

RESUMEN: El presente artículo trata sobre una investigación cualitativa realizada en la clínica de fisioterapia de la Universidade do Vale de Itajai/SC, con personas que tienen Enfermedad Pulmonar Obstructiva Crónica. El objetivo de este estudio fue identificar los sentimientos relacionados al problema de salud de esas personas. Para ello, se creó un grupo de convivencia con once pacientes y un familiar. A partir del análisis de los datos, surgieron tres categorías diferentes: la tristeza y el sufrimiento ocasionados por la enfermedad; las limitaciones e incapacidades físicas en el cotidiano, y el deseo de mejora en relación al cuadro clínico establecido por la enfermedad. Observamos que el grupo de convivencia es un espacio importante para las personas con enfermedades crónicas, en el sentido de provocar cambios de comportamiento, adquiriendo nuevos hábitos sanos de vida, sirviendo también como resorte propulsor para que estas personas puedan expresar sus sentimientos, contribuyendo así para mejorar su calidad de vida.
Endereço: Edilaine Kerkoski

R. Uruguai, 458, B1. 25A, sala 207

88.302-202 - Centro, Itajaí, SC.

E-mail: edilaine@univali.br
Artigo original: Pesquisa

Recebido em: 16/11/2006. Aprovação final: 12/04/2007. 


\section{INTRODUÇÃO}

Desde o início da década de 80, têm sido observadas mudanças expressivas no perfil da população mundial, destacando-se, em especial, o aumento na expectativa de vida e na proporção de idosos. Essas transformações implicam no surgimento de doentes crônicos, no contexto de uma população mais envelhecida, aumentando o período de morbidade com repercussões sociais, pessoais e de saúde. Para quem vive com a doença pode representar um prazo longo de sofrimento físico e psicológico, o que significaria uma piora da sua qualidade de vida. ${ }^{1}$

Existem doenças crônicas de grande impacto social e econômico, como a Doença Pulmonar Obstrutiva Crônica (DPOC), que merece algumas considerações especiais, muito embora, os dados epidemiológicos são restritos no país e, por esse motivo, as autoridades sanitárias não têm clareza do seu impacto na sociedade brasileira.

Nas últimas três décadas, a DPOC tornou-se uma importante causa de morbimortalidade e como conseqüência um problema de saúde pública. No mundo inteiro, dados epidemiológicos demonstram que milhões de pessoas sofrem desta enfermidade, e morrem prematuramente devido a suas complicações. Atualmente, a DPOC é a $12^{\mathrm{a}}$ doença mais prevalente no mundo e segundo a Organização Mundial da Saúde (OMS), será a quinta, no ano 2020. Da sexta causa de morte hoje, passará nesse mesmo período de tempo, para a segunda causa. ${ }^{2}$

É importante enfatizar que quando uma pessoa é diagnosticada com DPOC, ela deverá necessariamente modificar o seu estilo de vida, buscando uma melhor qualidade de vida. Seus hábitos deverão ser repensados, o uso de fumo, do álcool, o contato com poluentes, com fumantes, entre outros, deverão ser restritos e até mesmo abandonados. A partir do diagnóstico, a pessoa terá de conhecer profundamente a doença, suas complicações e principalmente o seu tratamento. Além disso, a pessoa deverá buscar novas relações sociais, trabalho compatível, novas modalidades de lazer e realizar ajustes financeiros. Isto se mostra bastante complexo, e a problemática gerada em torno dessa realidade tem conduzido os estudos, objetivando uma análise relacionada à qualidade de vida das pessoas com DPOC.

A conduta no atendimento das pessoas com DPOC é, sem dúvida nenhuma, a reabilitação pulmonar que requer uma ampla abordagem no tratamento e prevenção de complicações, exigindo por parte de profissionais que estes sejam capacitados para atender o paciente no seu todo, de modo a possibilitar apoio permanente, à medida que a doença avança. $O$ tratamento a ser dispensado envolve o esforço de uma equipe multiprofissional para conscientizar as pessoas com relação aos fatores de risco, principalmente no que se refere ao aconselhamento sobre a cessação do fumo a fim de prevenir a progressão da doença, no tratamento farmacológico para controle dos sintomas, no aconselhamento nutricional, na prática de exercícios físicos e, finalmente, no acompanhamento contínuo de enfermagem. ${ }^{3}$

Essa abordagem global no tratamento da DPOC estável é caracterizada por um avanço gradual do tratamento, que é dependente da gravidade da doença, e objetiva seu controle na tentativa de evitar inúmeras complicações e hospitalizações desses doentes.

Embora a educação seja importante em qualquer condição de saúde-doença, esta não tem sido considerada uma parte essencial da reabilitação pulmonar. ${ }^{4}$ É necessário destacar que educar pessoas acometidas pela doença sobre a DPOC, pode proporcionar não só a melhora das habilidades específicas, como também a condição de se lidar com a doença e a qualidade de vida. A educação encoraja a participação ativa da pessoa no cuidado da saúde e leva a uma melhor compreensão das alterações físicas e psicológicas que acontecem com a enfermidade crônica, ajuda as pessoas com DPOC e suas famílias a explorarem caminhos para lidar com as mudanças, tornando-as mais qualificadas no gerenciamento da doença e mais aderentes aos planos do cuidado. ${ }^{5}$

A educação em saúde deve envolver um trabalho buscando a participação dos indivíduos para assim obter e manter as metas de saúde. Para isso, faz-se necessário conhecer o ser humano e seu modo de vida, e buscar comprometê-lo nas respectivas ações de saúde. ${ }^{6}$

Neste sentido, práticas educativas desenvolvidas em grupos vêm se constituindo em uma prática cada vez mais freqüente e valorizada, pois permite ampliar conhecimentos, possibilitando as pessoas superarem suas dificuldades, adquirindo maior autonomia e o viver melhor com sua condição de saúde. ${ }^{7}$ O grupo de convivência visa o crescimento pessoal que abrange várias dimensões do processo de viver, a partir de necessidades comuns reveladas pelas pessoas, podendo manifestar um sentimento de união e vínculo se empenhando numa ação conjunta. ${ }^{8}$ 
Diante do exposto, esse estudo tem como objetivo desenvolver uma prática educativa visando identificar sentimentos dessas pessoas relacionados ao seu problema de saúde.

\section{METODOLOGIA}

Trata-se de uma pesquisa qualitativa realizada na Clínica de Fisioterapia da Universidade do Vale do Itajaí (UNIVALI), Campus da cidade de Itajaí - SC, no qual trabalham professores supervisores de estágio que orientam os alunos do último ano do Curso de Graduação em Fisioterapia e que prestam atendimento à comunidade em geral. A clínica ocupa uma área de 1.181,65 m², com espaços adequados aos pacientes portadores de qualquer tipo de deficiência e possui capacidade de realizar até 240 atendimentos diários, nas diversas áreas de atuação da Fisioterapia (pediatria, neurologia, reumatologia, ortopedia, traumatologia, respiratória, cardiovascular e ginecologia e obstetrícia). Semestralmente são realizados aproximadamente 900 atendimentos à pessoas com doenças pulmonares.

Os encontros do grupo de convivência aconteceram em salas do Curso de Fisioterapia, proporcionando conforto e privacidade para os participantes.

\section{Sujeitos da pesquisa}

Fizeram parte do estudo 11 pessoas com DPOC e um familiar. Cada uma dessas pessoas foi selecionada a partir dos seguintes critérios: ser portador de DPOC, estar em tratamento fisioterápico por no mínimo seis semanas na Clínica de Fisioterapia da UNIVALI, ter suas funções neuro-cognitivas preservadas, ter mais de 18 anos, ter interesse e disponibilidade em participar do estudo.

Participaram ainda do grupo de convivência junto com as pessoas com DPOC, duas pesquisadoras e um familiar, este último, por estar acompanhando sua mãe e manifestar interesse na sua participação.

\section{Coleta de dados}

Os encontros do grupo de convivência aconteceram uma vez por semana, durante os meses de outubro e novembro de 2006, num total de cinco. $\mathrm{O}$ agendamento foi realizado previamente no mês de setembro, tendo sido estabelecidas as datas dos respectivos encontros, a partir das possibilidades de cada um dos participantes. O tempo de duração médio de cada encontro foi de aproximadamente 120 minutos. As atividades desenvolvidas foram registradas no diário de campo e gravadas em fitas cassete com a autorização de todos os integrantes.

O grupo de convivência teve como proposta o desenvolvimento da educação em saúde, a fim de trabalhar os problemas que interferem na qualidade de vida de pessoas com DPOC. Para tanto, procurou-se realizar um processo reflexivo acerca dos problemas que interferem na qualidade de vida dessas pessoas, promovendo o compartilhar de saberes e experiências sobre o viver com a doença, procurando ainda, junto com estas pessoas, subsídios teóricos-reflexivos relativos a DPOC e conceitos de qualidade de vida que permitissem o desenvolvimento de capacidades potenciais para a construção de novas maneiras de viver com a doença crônica.

As fisioterapeutas foram moderadoras dos encontros e viabilizaram alguns temas de acordo com o interesse dos participantes, como: anatomia do sistema respiratório, fisiopatologia da DPOC, "re-treinamento" da respiração, nutrição e conservação de energia. Algumas técnicas e dinâmicas foram utilizadas para aquecer ou motivar os encontros, permitindo maior integração entre as pessoas, exposição dos seus conhecimentos e sentimentos. Além disso, foram também utilizadas algumas estratégias para a coleta de dados, a exemplo do encontro realizado sobre a representação do que é o viver com uma doença crônica pulmonar. Neste encontro, foi proposto ao grupo como dinâmica para a busca desta representação, a escolha de imagens em revistas. Após a escolha de uma ou mais figuras, ficando a critério de cada um, as mesmas foram coladas em um painel de papel pardo para a visualização de todos. Após a montagem do painel, cada um dos participantes justificou sua(s) escolha(s), acerca da imagem, e o que esta(s) representava(m) em sua vida convivendo com a doença pulmonar crônica.

\section{Análise dos dados}

Após as transcrições das fitas gravadas, os dados foram codificados, identificando as informações a respeito da questão qualidade de vida. Em seguida, foram estabelecidas as formações de categorias, segundo análise de conteúdo, ${ }^{9}$ que consiste num conjunto de expressões com características similares, 
consideradas interferindo (positiva ou negativamente) na qualidade de vida das pessoas com DPOC que integraram o grupo. Finalmente, foram identificadas três grandes categorias de análise: tristeza e sofrimento, limitações e incapacidades físicas no cotidiano e a esperança de melhora e persistência.

\section{Dimensão ética da pesquisa}

Considerando os aspectos éticos que permeiam o trabalho com seres humanos, foram levados em consideração os seguintes aspectos para a implementação da pesquisa, contemplando as determinações da Resolução No 196/96 do Conselho Nacional de Saúde: autorização e consentimento dos participantes para o registro, utilização e divulgação das informações obtidas no trabalho, através do Termo de Consentimento Livre e Esclarecido; explicação dos objetivos, metodologia e cronograma aos participantes e acerca das contribuições do estudo e da sua relevância social; garantia de "confidencialidade", privacidade e proteção da imagem dos participantes; respeito aos valores culturais, sociais, morais e religiosos e garantia da possibilidade de afastamento voluntário e espontâneo dos participantes. Este trabalho foi previamente encaminhado e analisado pelo Comitê de Ética em Pesquisa da UNIVALI e aprovado conforme parecer sob o $\mathrm{n}^{\circ} 842 / 06$.

\section{RESULTADOS E DISCUSSÃO}

A partir da análise dos dados resultantes da atividade desenvolvida junto com as pessoas com DPOC surgiram três categorias marcantes desta representação: a tristeza e sofrimento ocasionados pela doença; as limitações e incapacidades físicas no cotidiano; e o desejo de melhora em relação ao quadro clínico estabelecido pela doença. Embora marcadas como categorias distintas no intuito de facilitar a análise, estes aspectos na vida das pessoas com DPOC estão diretamente interligados. $\mathrm{Na}$ maioria das vezes, a tristeza e o sofrimento tem relação direta com as limitações físicas enfrentadas pela pessoa no seu cotidiano, restringindo as atividades simples que costumeiramente são realizadas junto aos familiares e de amigos. Por sua vez, estas limitações muitas vezes, acabam por se tornar "motivadoras”, no sentido do comprometimento na realização de tratamentos que possam devolver-lhe novas possibilidades de convívio social e desenvolvimento de atividades, no cotidiano doméstico.

\section{Caracterização dos participantes}

Dos onze integrantes do estudo, dois foram excluídos, por não poderem comparecer, uma vez que tiveram que ser internados em um hospital, por terem piorado seu quadro clínico. Os participantes tinham idade entre 37 e 81 anos, seis eram do sexo feminino e três do sexo masculino. Quanto ao grau de escolaridade, dois possuíam ensino superior, um ensino médio e seis ensino fundamental incompleto. Com relação ao tempo de doença, a média foi de 7,8 anos, variando de 3,6 a 10 anos. Todos os participantes realizavam tratamento com fisioterapia respiratória, com tempo médio de 2,4 anos.

\section{$1^{a}$ categoria: a tristeza e o sofrimento ocasio- nado pela doença}

A tristeza e o sofrimento se caracterizam por estados emocionais intrínsecos a todo e qualquer ser humano, privado de determinada satisfação pessoal e emocional. É uma reação do organismo quando o mesmo se depara profundamente com a sua fragilidade. A partir da fala das pessoas com DPOC percebe-se em muitos momentos, que esses sentimentos aparecem como "castigo" ou ao contrário, o oferecimento de oportunidade de "salvação", dando a conotação de aspectos de fé e religiosidade inerente à cultura e crenças individuais. Alguns percebem, também, que a tristeza e o sofrimento estão diretamente relacionados com as limitações impostas pela doença crônica pulmonar, porém, em muitas ocasiões, visualizam casos muito mais graves e complexos de outros, quando se deparam nas consultas de diferentes especialidades, ao observarem as salas de espera e através de conversas com outros pacientes nestes ambientes.

[... quem tem doença como a nossa, diz que a gente fica emocionada, fica triste. Eu achei uma figura que dir. a tristera faz bem também, será que é verdade? A gente tem momentos de tristera, mas a gente pode tirar proveito. Então achei sugestiva, ainda mais porque tristeza que é uma coisa ruim, dizem que faz bem. Quem sabe o sofrimento oferece salvação das almas, quem é católico acredita perfeitamente nisso (Céu).

[...] - olha, ainda não estou aleijado [referindo-se ao que seu pai falava quando the ofereciam ajuda durante sua melhora gradativa na recuperação de funções causadas por um AVC], então isso eu procuro imitar e usar pra mim. Eu ainda tenho alguma atividade, eu não faço coisas que en gostaria mais procuro fazer as que en posso e já não fico tão triste (Terra). 
[...] a gente pega aborrecimento, fica cada vez mais triste epior é (Sol).

Eu queria com essa foto aqui parecendo uma tristeza de uma doença crônica que a gente sempre tem né, mas com a esperança de nós ficarmos mais felizes na vida com ela (Sol).

A tristeza e o sofrimento, quando vivenciados por tempo prolongado, podem contribuir para o estabelecimento de quadros depressivos, com maior facilidade em idosos e doentes crônicos. A depressão é uma emoção universalmente experimentada por praticamente todos os indivíduos em alguma fase de suas vidas, muitas vezes relatadas como tristeza, saudade, angústia, desânimo, etc. Detectar a diferença entre as emoções "normais" do cotidiano e a doença depressão, talvez constitua no maior desafio para profissionais de saúde, principalmente para aqueles em que a formação nas ciências de saúde mental foi deficitária. ${ }^{10}$

Com o avanço dos trabalhos sobre prevalência de depressão e envelhecimento na literatura especializada, sabe-se que entre os pacientes com 65 anos ou mais, $17 \%$ a $30 \%$ podem apresentar sintomas depressivos que podem ou não estar associados com outras doenças.

Vários fatores têm sido identificados como predisponentes à depressão na vida tardia. Entre eles incluí-se a fragilidade da saúde, notadamente por maior prevalência de doenças como as cerebro-vasculares, infarto agudo do miocárdio, osteoartrose, doença pulmonar obstrutiva crônica e demência. ${ }^{10}$

A associação entre doenças crônicas e depressão ou transtornos de humor, tem sido freqüente na literatura e o impacto dessa comorbidade se traduz no aumento da utilização de recursos médicos, por conseguinte, dos custos associados ao tratamento. Tais pacientes são geralmente mais sintomáticos, têm maior comprometimento funcional na vida diária, maiores dificuldades na adesão ao tratamento farmacológico e mudanças de hábitos de vida considerados importantes para a evolução do tratamento como interrupção do tabagismo e dieta. ${ }^{11}$

Reforçando esta mesma idéia, conforme descrito no Manual Diagnóstico e Estatístico de Transtornos Mentais, DSM-IV-TR ${ }^{\mathrm{TM}},{ }^{12}$ o Transtorno Depressivo Maior (TDM), classificado na categoria "transtorno de humor", pode estar associado com condições médicas gerais crônicas. Até $20-25 \%$ dos indivíduos com certas condições gerais desenvolvem TDM durante o curso da doença crônica. Como resultado o tratamento da condição médica geral é mais complexo e o prognóstico menos favorável quando um TDM está presente.

Como repercussões comuns ao indivíduo que possui TDM podem ocorrer alterações do apetite ou peso, sono, atividade psicomotora caracterizado por agitação ou retardo psicomotor, diminuição da energia, sentimentos de menos valia ou culpa, dificuldade para pensar, concentrar-se ou tomar decisões ou pensamentos recorrentes sobre morte. ${ }^{12-14}$ É importante perceber que algumas destas manifestações como alterações do apetite ou peso, sono, diminuição da energia, sentimentos de menos valia ou culpa, são comuns à pessoa com DPOC e que podem estar sendo reforçadas por transtornos depressivos, colocando diretamente em risco o tratamento e melhora do indivíduo.

As alterações do sono ocorrem devido à dificuldade de manter-se na posição horizontal pela dificuldade respiratória imposta, tendo que dormir muitas vezes na posição semi-sentada para melhor conforto respiratório. Também há dificuldade de chegar a todas as fases do sono, principalmente o sono profundo, pois é comum acordar várias vezes durante a noite, permanecendo a sensação de cansaço no dia seguinte devido à falta do sono reparador.

Diminuição de energia, cansaço e fadiga são comuns devido ao importante gasto energético intrínseco na pessoa com DPOC. O indivíduo pode relatar fadiga persistente sem esforço físico e, mesmo as tarefas mais leves, parecem exigir um esforço substancial. Este fato é constatado na atividade com o grupo, a partir de suas falas e representações, apontando a segunda categoria firmada que são as limitações e incapacidades físicas no cotidiano.

\section{$2^{a}$ categoria: as limitações e incapacidades físicas no cotidiano}

A incapacidade para realização de tarefas consideradas simples e corriqueiras passa a ser extremamente desgastante para estas pessoas, virando fonte de sofrimento e angústia para a pessoa com DPOC. O surgimento de sentimentos de menos valia, de invalidez, de dependência e a da vergonha de tossir, são retratados conforme os depoimentos das pessoas com DPOC ao explicarem o que as levou a escolha de determinadas figuras que representasse o viver com uma doença crônica, no caso a doença pulmonar obstrutiva crônica: [...] épequenininha a foto, é uma pessoa tossindo, eu fico muito envergonbado de tossir, 
minha mulher disse que eu tusso muito alto, tem que tossir mais baixo, não dá, é uma coisa que não dá pra controlar, que nem a falta de ar, então essa foto me chamou a atenção, vou colocar aqui perto da foto do cigarro (Terra).

[...] eu também tenho vergonba de tossir, incomoda os outros, não gosto (Mar).

[...] essa daqui épor que eu me sinto sufocada às vezes, não sei se vocês se sentem, parece que fica tudo trancado, me dá uma agonia, essa aqui eu tirei porque eu tenho dificuldade de andar muito rápido, correr, fazer algum exercicio muito rápido. Essa aqui eu tirei porque eu tenho dificuldade de fazer as atividades de casa, me dá muita canseira, muita falta de ar, tenho dificuldade de varrer, de passar pano, a poeira faz. mal pro pulmão (Vento).

[...] essa daqui é o que eu sinto vontade de fazer e não posso, que éparticipar das brincadeiras com a bisneta que eu fico muito cansado e ela quer que eu pule corda, ela quer que eu ande atrás da bicicleta ai não dá... Então isso daqui é uma dificuldade que eu tenho que me deixa triste. Essa daqui é uma dupla face, as dificuldades que eu tenho de não poder brincar e namorar, ta difícil. Então como é que eu faço, vou botar essa daqui pra frente [foto de um casal se beijando] essa é mais bonita (Terra).

Nessa foto tem uma pessoa que está muito triste. É muito difícil viver com a doenca, mas primeiro tem que tentar né, mas é dificil e é muito triste, a gente pensa assim: - Oh, meu Deus en vejo velhinha de 90 anos, tem uma velhinha perto da minha casa, ela tem 85 anos, ela planta, ela varre a calcada que é grande, ela vai correndo pegar o ônibus sożinha, eu não consigo fazer isso, é muito triste, tenho que ter sempre uma pessoa, eu tenho dificuldade pra entrar num carro, pra caminhar, pra fazer as coisas em casa (Lua).

[...] é muito difícil, a gente não se conforma, tantas pessoas que andam correndo de lá pra cá e a gente não pode, se eu ando um pouquinho demais falta ar, não posso fazer nada falta ar, não posso passear porque ta me incomodando, é horrivel, é horrivel, a gente pensa tanta coisa, até besteira... a gente toma remédio nada adianta, nada adianta, né, vou no médico ele diz. isso não tem cura, tem que conviver com isso mesmo, aí eu fico triste, né, en queria ficar boa (Mar).

Nas falas acima, aparecem claramente as sobreposições entre as categorias tristeza e sofrimento e limitações e incapacidades físicas no cotidiano problema, referido anteriormente como situações que retro-alimentam negativamente a vida das pessoas com DPOC. O movimento e a atividade física são inerentes ao ser humano, e a experimentação de limitações aos movimentos simples como caminhar e as repercussões nas esferas familiar, social, sexual, etc são extremamente angustiantes por si só. Porém, tem-se aqui a soma dos fatores complicadores destas limitações serem causadas por doenças crônicas degenerativas, ou seja, sem grandes perspectivas de melhora a ponto de poderem desenvolver atividades consideradas normais a qualquer homem. Esta retro-alimentação negativa fica clara quando notase que grande parte da tristeza declarada é devido ao "eu sinto vontade de fazer e não posso" causando ainda mais tristeza e sofrimento, reforçando as chances do aparecimento de quadros depressivos, conforme já discutimos.

Atividade física é todo e qualquer movimento corporal produzido pela musculatura esquelética (voluntária) que resulta em gasto energético acima dos níveis de repouso. ${ }^{15}$ No caso do paciente com DPOC, as limitações e incapacidades físicas se referem às atividades consideradas leves, que são aquelas nas quais a demanda energética situa-se até $3 \mathrm{METs}$ (gasto energético consumido). São atividades que podem ser realizadas por longo período de tempo e que requerem um mínimo esforço. São as atividades que trabalham menos de $60 \%$ da freqüência cardíaca máxima. ${ }^{15}$

Aparece com muita freqüência, a fala positiva em relação à fisioterapia e a melhora obtida com a adesão ao tratamento. O exercício físico é uma das formas de atividade física planejada, estruturada, sistemática, efetuada com movimentos corporais repetitivos, a fim de manter ou desenvolver um ou mais componentes da aptidão física como a agilidade, coordenação, equilíbrio, força muscular, flexibilidade, resistência, dentre outros. A fisioterapia especificamente na abordagem da pessoa com DPOC, tem como objetivos o condicionamento físico, ganho de força muscular global e treinamento da respiração no intuito de diminuição da dispnéia e melhor conforto respiratório.

Com a melhora da condição clínica e a melhor qualidade de vida alcançada pelas pessoas com DPOC que estão realizando fisioterapia e participando deste grupo convivência, surge a terceira e última categoria mapeada nesta pesquisa, a esperança de melhora e persistência.

\section{$3^{a}$ categoria: a esperança de melhora e per- sistência}

Além dos depoimentos feitos por estas pessoas neste sentido, observa-se que houve também uma maior adesão ao tratamento fisioterápico, com a diminuição de faltas e dedicação durante a sessão. Assim como maior adesão aos tratamentos necessá- 
rios: maior cuidado na alimentação, o uso correto de medicamentos e manter-se ativo (do ponto de vista físico e cognitivo) o máximo possível nas atividades de vida diária são itens reforçados pelo grupo como aspectos positivos, desencadeados a partir do tratamento e reforçados pelo convívio enquanto grupo.

Essa aqui eu tirei porque en queria uma foto que lembrasse a juventude nova com a mão pronta para pegar um projeto mais pra frente, nós temos que ter esperança nos novos pra nós termos alegria na nossa juventude velha. Essa aqui dá alegria pra todo mundo, é o violeiro (música), ele dá alegria e no mesmo tempo dá esperança para a gente viver melhor. E agradeço nossa presença aqui, porque tenho esperança de melhorar mais porque a nossa saúde é o nosso principal (Sol).

[...] aqui tão distante, maldito cigarro, maldito [figura de um homem fumando]. Achei aqui figura de fisioterapeuta, graças a Deus existe neste mundo gente que tem essa profissão com grande habilidade, que cuidam de nós. Agora quando a gente vem fazer fisioterapia achei uma figura bem estar, a gente sai daqui bem melhor do que quando a gente chegou, não é verdade? Então o bem-estar, é uma figura, alguém que está feliz. da vida tranqüilo. Essa aqui é uma piscina, então é muito bom me lembra do esporte sobretudo a bidroginástica, que é importante e aqui a gente encontra uma piscina com água quentinha eu tenho ido lá (Céu).

[...] até os 18 anos quando eu tive a terceira pneumonia, era só xaropinho assim, como falei tinha primos médicos e não foi feito um tratamento sistemático. Eu fui a Campinas lá tinha um instituto pneumológico e o médico lá me deu tratamento prolongado, em fevereiro até julho tomando tiaminose, fortificante, xarope, tinha penicilina também, depois en voltei pra casa, fui morar em Londrina, eu estudei. Agora sempre nas crises, nunca senti falta de ar, sempre era o catarro, tosse, e de vez. em quando a febre que dava o alarme, agora aqui que estou tendo esta oportunidade de fazer a fisioterapia para melhorar o modo de vida da gente, estou muito contente (Céu).

[...] estou muito contente de ta aqui com você, a gente ta aprendendo mais né (Estrela).

Nota-se no grupo, a identificação por eles próprios, da consciência das limitações que cercam a doença pulmonar obstrutiva crônica, bem como os fatores complicadores e o prognóstico que se delineia para cada um. Porém, percebe-se que a melhora e a estabilização do quadro clínico tem sido motivadora. $\mathrm{O}$ resgate desta motivação se reflete em vários sentidos, como descrito anteriormente, mas principalmente se reflete na maior alegria e felicidade na vida destas pessoas e suas famílias. Este aspecto deve ser o principal, e mais importante a ser considerado, quando levamos à frente, propostas de educação em saúde como esta. Em oposição ao tecnicismo que predomina atualmente nas práticas de saúde, percebemos que muitas das técnicas e tecnologias utilizadas em prol do paciente não surtem efeitos, talvez porque estejam, na maioria das vezes, focadas na doença e não no doente.

\section{CONSIDERAÇÕES FINAIS}

Entender acerca da vida cotidiana que se passa com o doente é seguramente o melhor caminho para a aproximação, a criação do vínculo e a adesão ao tratamento proposto ao doente. Cada vez mais, as práticas em saúde devem considerar o sujeito e seu meio como atores principais e norteadores na eleição e estabelecimento de tratamentos para cada indivíduo.

Quando nos propomos a trabalhar com educação em saúde, muitas vezes não percebemos a complexidade de fatores envolvidos neste processo. Educar parte do pressuposto de que algo é ensinado e apreendido, de que existe um sujeito facilitador na aprendizagem de algo (o educador) e outro que apreende informações. Educar pressupõe de quem educa, estar aberto também ao aprendizado com aquele(s) com quem propõe educar, ser humilde e reconhecer que todos são educadores e aprendizes. $\mathrm{O}$ apreender significa segurar, prender, pegar, assimilar mentalmente, entender, compreender, agarrar. Não se trata de um verbo passivo.

Para apreender é preciso agir, exercitar-se, informar-se, tomar para si, apropriar-se, entre outros fatores. $\mathrm{O}$ aprender significa tomar conhecimento, reter na memória, receber informação de algo, ou seja, conota passividade e superficialidade no processo, não sendo gerador de mudanças. ${ }^{16}$ Esta simples modificação semântica tem impacto importante em nossas ações, onde devemos refletir de imediato se com a proposta de educação em saúde desejamos que os sujeitos, aqui as pessoas com DPOC, simplesmente aprendam ou apreendam informações capazes de lhes repercutir de forma positiva e eficaz. Por fim, o processo de educação, e aqui reforçamos a educação em saúde, só se concretiza ou se mostra efetivo quando implica em mudança de comportamento do indivíduo que apreende o que lhe foi ensinado. Esta mudança de comportamento voltada para cuidar de si, perceber-se, prevenir-se a partir de atitudes simples no dia-a-dia e seu impacto positivo na qualidade de vida das pessoas é a solidificação da proposta educativa em saúde. 
Este estudo nos possibilitou uma visão mais ampliada da prática assistencial e a percepção que a educação em saúde, através de grupos de convivência pode contribuir para melhorar a qualidade de vida de pessoas com doença pulmonar obstrutiva crônica. $\mathrm{O}$ trabalho em grupo é uma alternativa que traz resultados satisfatórios pela ação conjunta das pessoas que dele participam no compartilhar de saberes e experiências buscando um novo olhar para um viver mais saudável.

\section{REFERÊNCIAS}

1 Lessa I. O adulto brasileiro e as doenças da modernidade: epidemiologia das doenças crônicas não-transmissíveis. São Paulo (SP): Hucitec/Abrasco; 1998.

2 Yaksic MS, Tojo M, Cukier A, Stelmach R. Prolife of a brazilian population with severe chronic obstructive pulmonary disease. Jour. Pneumol. 2003 Mar-Abr; 29(2): 64-8.

3 World Health Organization. Gold: global strategy for the diagnosis, management, and prevention of chronic obstructive pulmonary disease. Washington (EUA): NHLBI/WHO workshop report; 2001.

4 Mahler DA. Pulmonary rehabilitation. Chest 1998 May; 113 (4): 263S-268S.

5 American Thoracic Society. Pulmonary Rehabilitation. American Jour. Respiratory Critical Care Med. 1999 May; 159 (5): 1666-82.

6 Briceño-Léon R. Siete tesis sobre la educación sanitaria para la participación comunitaria. Cad. Saúde Públ. 1996 Jan-Mar; 12 (1): 7-30.
7 Silva DMGV, Francioni FF, Natividade MSL, Azevedo M, Sandoval RCB, Di Lourenzo VM. Grupos como possibilidade para desenvolver educação em saúde. Texto Contexto Enferm. 2003 Jan-Mar; 12 (1): 97-103.

8 Trentini M, Gonçalves LHT. Pequenos grupos de convergência: um método no desenvolvimento de tecnologias na enfermagem. Texto Contexto Enferm. 2000 Jan-Abr; 9 (1): 63-78.

9 Bardin L. Análise de conteúdo. 3a ed. Lisboa (PT): Ed. 70; 2004.

10 Freitas EV. Tratado de geriatria e gerontologia. Rio de Janeiro (RJ): Guanabara Koogan; 2002.

11 Moreno DH, Bernik M, Mattos P, Cordás TA. Recuperação em depressão. São Paulo (SP): Editora Livre; 2003.

12 Associação Americana de Psiquiatria. Manual diagnóstico e estatístico de transtornos mentais: DSMIV-TR ${ }^{\mathrm{TM}}$ [Dornelles C, tradutor]. 4a ed. Porto Alegre (RS): Artes Médicas; 2002.

13 Pickles B. Fisioterapia na terceira idade. 2a ed. São Paulo (SP): Santos; 2000.

14 Stoppe Júnior A, Louzã Neto MR. Depressão na terceira idade: apresentação clínica e abordagem terapêutica. São Paulo (SP): Lemos; [19- ].

15 Mazo GZ, Lopes MA, Benedetti T B. Atividade física e o idoso: concepção gerontologica. Porto Alegre (RS): Sulina; 2001.

16 Anastasiou LGC. Alves LP. Processos de ensinagem na universidade: pressupostos para as estratégias de trabalho em aula. Joinville (SC): Ed. UNIVILLE; 2004. 